\title{
TLR signalling and activation of IRFs: revisiting old friends from the NF- $\mathrm{B}$ pathway
}

\author{
Paul N. Moynagh
}

Department of Pharmacology, Conway Institute of Biomolecular and Biomedical Research, University College Dublin, Belfield, Dublin 4, Ireland

Toll-like receptors (TLRs) are crucially important in the sensing of infectious agents. They serve to recognize pathogen-associated molecules and respond by triggering the induction of specific profiles of proteins that are tailored to the successful removal of the invading pathogens. The induction of TLR-responsive genes is mediated by the activation of transcription factors, and most interest has focussed on NF- $B$, a transcription factor that is universally used by all TLRs. However, there has recently been a burgeoning effort to increase our appreciation of the importance of members of the interferon-regulatory factor (IRF) family in TLR signalling. This review will discuss the most recent findings relating to the regulation of IRF activity by TLRs and will highlight the rapidly increasing complexity of TLR signalling pathways.

\section{Introduction}

Human Toll-like receptors (TLRs) act at the hostpathogen interface and are the earliest surveillance systems for primary infection by pathogens. To date, 11 TLRs have been identified and demonstrated to recognize a variety of pathogen-associated molecular patterns (PAMPs). The engagement of TLRs by pathogenic components results in the induction of specific gene expression profiles that are suited to ensuring efficient removal and destruction of the invading pathogen. The repertoire of TLR-responsive genes range from proinflammatory cytokines and interferons (IFNs) (which orchestrate innate immunity) to co-stimulatory molecules (which promote T-cell activation and specific immunity). The capacity of TLRs to trigger the induction of tailored profiles of genes is underpinned by their ability to activate a variety of transcription factors. Much interest has focussed on NF- $\mathrm{NB}$, a transcription factor that is activated by all TLRs. However, many recent studies have highlighted specific members of the IFN-regulatory factor (IRF) family as crucial factors in the induction of TLR-responsive genes. This review will give an overview of the role of IRFs in TLR signalling and recent advances in the identification of key signalling molecules used by TLRs for the activation of IRFs.

\footnotetext{
Corresponding author: Moynagh, P.N. (P.Moynagh@ucd.ie).
}

Available online 11 July 2005
TLR signal transduction and activation of NF- $\mathrm{KB}$

Recent studies in relation to TLR signal transduction and IRF activation have emphasized dual roles for signalling molecules, with respect to triggering activation of both the $\mathrm{NF}-\kappa \mathrm{B}$ and IRF pathways. Indeed, some of the key signalling molecules that mediate TLR activation of IRFs were initially discovered based on their ability to activate NF- $\mathrm{B}$. Thus, an initial overview of the various TLR signalling pathways that activate $\mathrm{NF}-\kappa \mathrm{B}$ forms a suitable context for discussion of recent advances in TLR activation of IRFs.

Myd88-dependent signalling and activation of NF- $\mathrm{B}$ TLRs use many of the same signalling components as the IL-1 receptor (IL-1R) as a result of their intracellular regions sharing a conserved Toll-IL-1R (TIR) domain [1]. The proximal signalling events subsequent to TLR engagement by an appropriate ligand have been well characterised (Figure 1). With the exception of TLR3, all TLRs recruit the TIR domain-containing adaptor molecule myeloid differentiation factor 88 (Myd88) [2]. Myd88 then associates with members of the IL-1R-associated kinase (IRAK) family (IRAK-1-4) [1]. IRAK-1 is recruited to Myd88 within a complex with another protein termed Tollinteracting protein (Tollip) [3]. The IRAK-Myd88 association triggers hyperphosphorylation of IRAK-1 by itself and other kinases, such as IRAK-4 [4,5], which leads to its dissociation from Myd88 and Tollip and its interaction with the downstream adaptor tumour necrosis factor (TNF) receptor-associated factor 6 (TRAF-6) [3]. TRAF-6 is an ubiquitin ligase [6,7] and activates transforming growth factor- $\beta$ (TGF- $\beta$ )-activating kinase (TAK1) [8], which in turn promotes downstream activation of the I $\kappa$ B kinases (IKK), IKK $\alpha$ and IKK $\beta$. The IKKs directly phosphorylate members of the inhibitory $\mathrm{I} \kappa \mathrm{B}$ family, which normally sequester $\mathrm{NF}-\kappa \mathrm{B}$ in an inactive form in the cytosol [9]. The phosphorylation of the I $\kappa$ B proteins represents a signal for polyubiquitination followed by their degradation by the $26 \mathrm{~S}$ proteosome and this enables the translocation of $\mathrm{NF}-\kappa \mathrm{B}$ to the nucleus, where it induces a plethora of genes encoding proinflammatory proteins and co-stimulatory molecules [10,11].

Myd88-independent signalling and activation of NF- $\kappa$ With the exception of TLR3, the above pathway is used universally by all TLRs in activating NF- $\mathrm{TB}$ and has been 


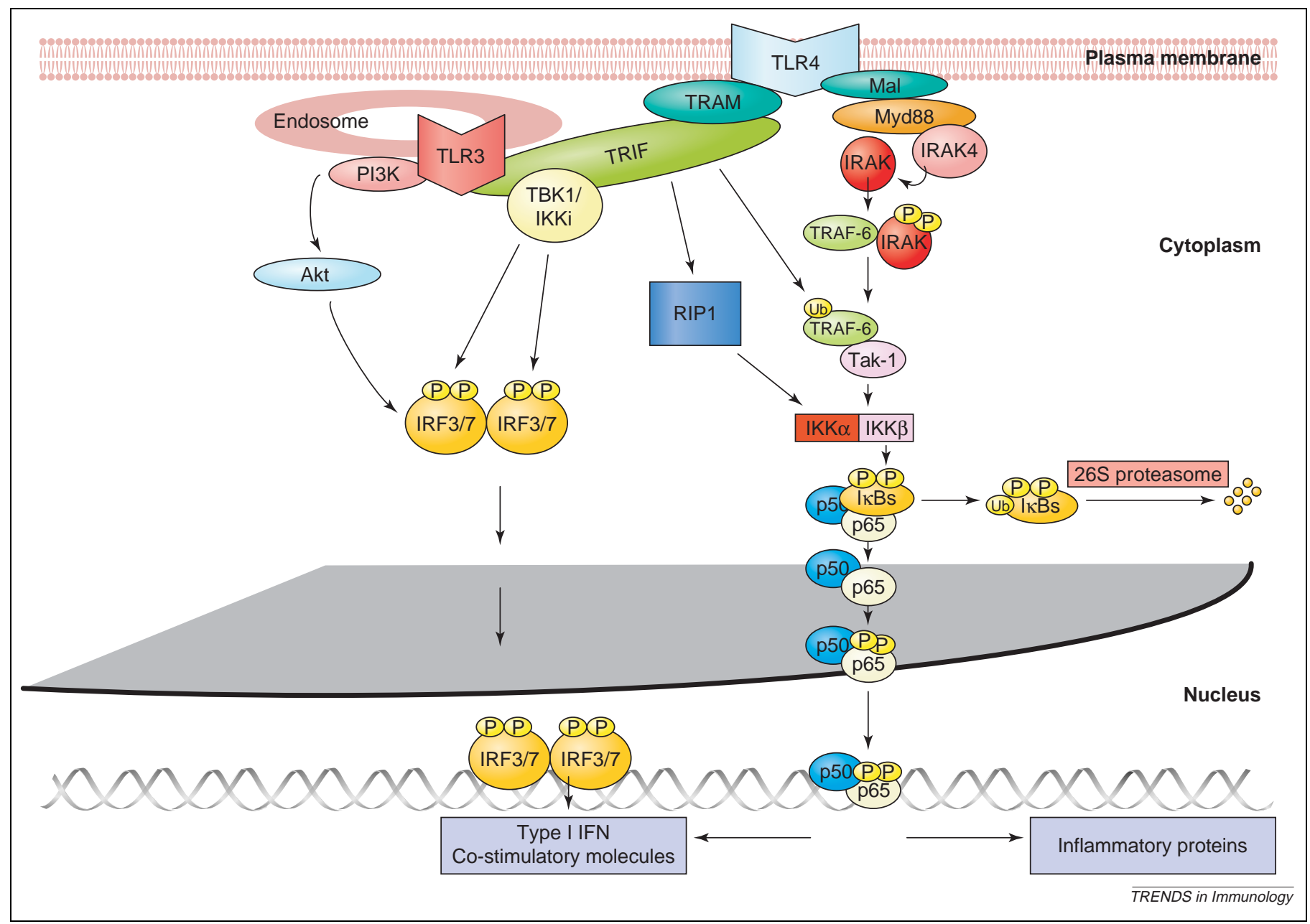

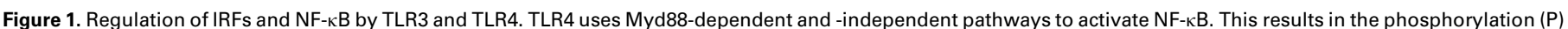

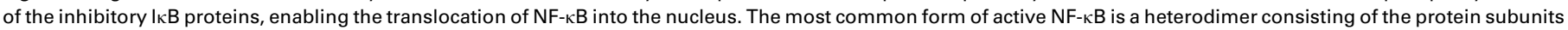

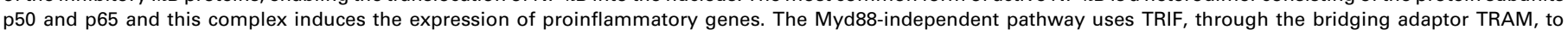

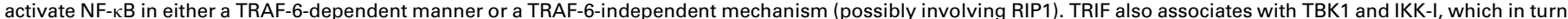

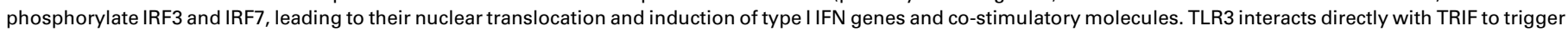
these Myd88-independent pathways. In addition, TLR3 interacts with PI3K and activates Akt, leading to further phosphorylation and maximal activation of IRF3 and IRF7.

termed Myd88-dependent signalling [12]. TLR3 signals in a Myd88-independent manner. Interestingly, the TLR4 ligand lipopolysaccharide (LPS) can still trigger activation of NF-кB in Myd88-deficient mice, albeit with delayed kinetics compared with wild-type cells, whereas most other TLRs are completely ineffective at activating NF- $\mathrm{KB}$ in these mice [13]. Although Myd88-deficient mice lose their ability to produce proinflammatory cytokines, such as TNF, IL-1, IL-6 and IL-12 p40, in response to LPS, they retain their capacity to produce IFN-inducible genes and co-stimulatory molecules and their dendritic cells (DCs) mature in response to LPS [14,15]. These studies define two arms of the LPS signalling pathway: a Myd88-dependent component that promotes fast activation of $\mathrm{NF}-\mathrm{\kappa B}$ and induction of proinflammatory molecules and a Myd88-independent pathway (also used by TLR3) that mediates slow activation of NF- $\mathrm{KB}$ and induction of IFN-inducible genes and costimulatory molecules (Figure 1).

A second TIR domain-containing adaptor protein was identified by two independent groups, and was named MyD88 adaptor-like (Mal), also known as TIR domaincontaining adaptor protein (TIRAP) $[16,17]$. However, the generation of Mal (TIRAP) knockout mice showed that this adaptor is not responsible for Myd88-independent signalling and instead acts as a bridging adaptor between Myd88 and TLR4 and TLR2 [18,19]. Subsequent studies identified TIR domain-containing adaptor-inducing IFN- $\beta$ (TRIF), also known as TIR-containing adaptor molecule (TICAM)-1, as the key regulator of the Myd88-independent pathway. TRIF-knockout mice, or mice with a frame shift mutation resulting in a truncated form of TRIF, fail to show Myd88-independent activation of NF- $\mathrm{BB}$ and are non-responsive to LPS or the TLR3 ligand poly(I:C), with respect to the expression of IFN-inducible genes [20,21]. TRIF interacts directly with the TIR domain of TLR3 [22] and mediates signalling, whereas another TIR domaincontaining adaptor TRIF-related adaptor molecule (TRAM), also known as TICAM-2, acts as a bridging adaptor between TLR4 and TRIF [23]. TRIF interacts with TRAF-6, the downstream adaptor with a previously described role in the Myd88-dependent pathway. However, there are contrasting reports on the importance of TRAF-6 for mediating NF- $\mathrm{KB}$ activation by TRIF. The mutation of the TRAF-6-binding site in TRIF reduces its ability to activate NF- $\mathrm{B}[24,25]$ and Jiang et al. [24] have demonstrated that TLR3-induced NF- $\mathrm{BB}$ is completely 
abolished in embryonic fibroblasts from TRAF-6-deficient mice. By contrast, Gohda et al. [26] have reported that TLR3 activation of NF- $\mathrm{KB}$ is normal in TRAF-6-deficient macrophages. The discrepancy might be owing to the cell type-specific roles of TRAF-6 in TLR3 signalling or functional redundancy of TRAF-6 in macrophages. Indeed, TRIF can also associate with the kinase receptor-interacting protein 1 (RIP1) and can activate NF- $\mathrm{KB}$ [27]; this might facilitate TRAF-6-independent activation of NF- $\kappa \mathrm{B}$ by TLR3 in macrophages.

\section{Activation of IRF3 and IRF7 by TLR3 and TLR4}

The initial discovery of Myd88-independent pathways in TLR signalling was predominantly based on the findings that TLR3 and TLR4 ligands retain their ability to trigger the expression of type I IFN and IFN-inducible genes in Myd88-deficient mice [13,28]. Type I IFNs are encoded by one $I F N-\beta$ gene and multiple $I F N-\alpha$ genes. They comprise potent antiviral molecules and it is easy to appreciate the value of having virally derived double stranded (ds)RNA induce their expression through TLR3. However, type I IFNs also have an important role in bridging innate and adaptive immunity by mediating the induction of costimulatory molecules on antigen-presenting cells in response to pathogen-associated molecules, such as LPS [29]. Much interest has focussed on the regulatory pathways that control the expression of type I IFNs.

In response to viral infection, IRF3 and IRF7 are the most important transcription factors that regulate type I IFN expression (reviewed in Ref. [30]). Prior to infection, IRF3 is normally expressed at high constitutive levels in most cells, whereas IRF7 is present at much lower levels. Indeed, the central dogma is that IRF3 is responsible for the early wave of IFN- $\beta$ gene induction. IRF3 shuttles into the nucleus by virtue of its nuclear localisation sequence; however, it also possesses a nuclear export signal that precludes high steady state nuclear levels of IRF3. Viral infection triggers phosphorylation of a serine/threonine cluster in the C-terminal region of IRF3. This causes a change in conformation, revealing an IRF-association domain and DNA-binding domain, enabling the dimerization of IRF3 and binding to IRF3 motifs in relevant promoters. The C-terminal phosphorylation of IRF3 also facilitates its association with the co-activators CBP [CREB (cAMP response element-binding protein)-binding protein] and p300 and these interactions prevent IRF3 export to the cytoplasm. IRF3 primarily activates the IFN- $\beta$ promoter with the co-operation of other transcription factors, especially NF- $\kappa \mathrm{B}$. Once IFN- $\beta$ is produced, it subsequently activates a transcription factor complex, known as IFN-stimulated gene factor 3 (ISGF3), comprising STAT1 (signal transducer and activator of transcription1), STAT2 and IRF9. ISGF3 in turn induces the expression of IRF7 and this is subject to virus-induced phosphorylation in a manner similar to IRF3. This leads to activation of IRF7 and its stimulation of $I F N-\alpha / \beta$ promoters. The induction of type I IFNs establishes a positive feedback loop, ensuring high-level expression of type I IFN and IFN-inducible genes.

Because TLR3 and TLR4 induce type I IFNs by mechanisms independent of Myd88, recent attention has focussed on the mechanism by which TRIF induces type I IFN and more specifically its ability to promote activation of IRF3 and IRF7. The need for complete delineation of this mechanism has become more pressing, with recent reports showing that viruses have developed immune evasion strategies including the sequestration of TRIF by the viral protein A46R and the cleavage of TRIF by the viral protease NS3/4a. These strategies are discussed in more detail by Schröder and Bowie, also in this issue [31].

\section{TBK1 and IKK-I activate IRF3 and IRF7}

The engagement of TLR3 and TLR4 by poly(I:C) and LPS, respectively, promotes phosphorylation of IRF3 and the induction of type I IFN genes [14,32,33]. An intense research effort culminated in key reports identifying two kinases responsible for this phosphorylation [34,35]. Both kinases are related to the IKK kinases and are inducible IKK (IKK-i) (also known as IKKe) and TRAF family member-associated NF- $\kappa \mathrm{B}$ activator (TANK)-binding kinase 1 (TBK1) [also called NF- $\mathrm{NB}$-activating kinase (NAK) or TRAF-2-associated kinase (T2K)]. Although the overexpression of both forms is sufficient to activate NF- $\kappa \mathrm{B}$, this is independent of the phosphorylation and degradation of $I \kappa \mathrm{B}$ proteins and is more related to the phosphorylation and increased transactivation of the $\mathrm{NF}-\kappa \mathrm{B}$ subunit p65 [36,37]. However, there is no definitive report defining the physiological role of these kinases in NF- $\kappa \mathrm{B}$ signalling. Fitzgerald et al. [34] showed that IKK-i and TBK1 mediate TLR3 and TRIF activation of IRF3 (Figure 1). Both IKK-i and TBK1 associate with TRIF and a recent study has suggested that the TANK family protein NAK-associated protein 1 (NAP1) might facilitate the interaction of both kinases with TRIF [38]. The work of Fitzgerald et al. [34] suggests that there is a TLR3-TRIF-IKK-I (TBK1)-IRF3 signalling axis. However, the study did not address the issue of whether IRF3 is a direct substrate for IKK-i and TBK1. A simultaneous study by Sharma et al. [35] demonstrated that IKK-i and TBK1 can directly phosphorylate IRF3 at its C-terminal serine/threonine cluster and that this is sufficient to induce nuclear translocation and DNA binding of IRF-3. This has been independently confirmed [39]. Furthermore IKK-i and TBK1 also phosphorylate and activate IRF7 similarly [35].

To study the in vivo function of IKK-i and TBK1, relevant knockout mice were generated [37,40]. Deficiency in TBK1 causes TNF-mediated liver degeneration, resulting in embryonic lethality [37]. However, embryonic fibroblasts were generated from $T B K 1^{-1-}$ mice, stimulated with TLR3 and TLR4 ligands and characterised relative to wild type cells, in relation to IRF3 activation and induction of type I IFNs and IFN-inducible genes. There was severe impairment of the activation and nuclear translocation of IRF3 and induction of IFN- $\alpha$, IFN- $\beta$ and IFN-inducible genes, such as RANTES and IP10 (IFN- $\gamma$-inducible protein 10), in response to both TLR ligands $[39,40]$. Such defective TLR3 and TLR4 signalling is also evident in bone marrow-derived macrophages derived from $T B K 1^{-1-} T N F R 1^{-1-}$ mice [41], further emphasizing the crucial role of TBK1 in promoting IRF3-dependent gene expression. The in vivo role of 
IKK-i in IRF3 signalling is less clear than that for TBK1. Embryonic fibroblasts from $I K K-i^{-1-}$ mice showed normal responses to dsRNA and LPS with respect to IRF3 activation and induction of type I IFNs. However, IKKi $i^{-1-} T B K 1^{-1-}$ mice fail to express IFN genes or IFN-inducible genes in response to dsRNA, and thus IKK-i might account for the low residual expression of these genes still observed in $T B K 1^{-1-}$ cells [40]. Furthermore, reconstitution of $T B K 1^{-1-}$ cells with IKK-i can compensate partially for the lack of TBK1, with respect to IRF3 activation and induction of IFN- $\beta$. This suggests at least some degree of functional redundancy between the two kinases in activating IRF3. However, such redundancy might only be relevant in specific cell types because TBK1 is produced constitutively in all cells, whereas IKK-I production is more restricted [41].

It is worth noting that the activation of $\mathrm{NF}-\kappa \mathrm{B}$ is normal in response to dsRNA and LPS in $T B K 1^{-1-}$ and $I K K-i^{-1-}$ cells, demonstrating that TBK1 and IKK-i have specific roles in mediating the activation of IRF3 and not NF- $\kappa \mathrm{B}$ in TLR3 and TLR4 pathways. Thus, a picture emerges of a bifurcation into two distinct signalling pathways downstream of TRIF (Figure 1). TRIF can interact with TRAF-6, leading to classical activation of $\mathrm{NF}-\kappa \mathrm{B}$ and the induction of proinflammatory proteins, such as IL-1, IL-6 and TNF, whereas mutation of the TRAF-6-binding site in TRIF fails to affect its ability to activate IRF3. However, TRIF can also interact with TBK1 to promote the activation of IRF3 and IRF7, leading to the induction of type I IFN and IFN-inducible genes. These two TRIF-mediated pathways are triggered by TLR3 (through its direct interaction with TRIF), and TLR4 by its indirect association with TRIF (through the bridging adaptor TRAM) $[42,43]$. The concomitant activation of NF- $\kappa$ B and IRF3 by TRIF is of biological significance because the activation of the promoters of some genes, such as $I F N-\beta$, requires the functional cooperation of these two transcription factors [44].

The downstream mechanism by which TRIF ultimately activates IRF3 might differ between the TLR3 and TLR4 pathways because dsRNA causes phosphorylation of the key serine residue 396 (Ser396) in the C-terminal region of IRF3, whereas LPS fails to induce phosphorylation at this site [33]. However, a more recent report has questioned the importance of Ser396 as the primary phosphoacceptor site and instead provides data to indicate that Ser386 is the crucial residue that undergoes inducible phosphorylation, thus leading to IRF3 activation [45]. This study provides a more satisfying mechanistic basis for TRIF activation of IRF3 because both TBK1 and IKK-I can phosphorylate IRF3 at Ser386 and the engagement of TLR3 and TLR4 by dsRNA and LPS leads to phosphorylation of this residue.

TLR3 has been shown recently to be phosphorylated on two key tyrosine residues in response to its engagement by dsRNA and this leads to the recruitment of phosphatidylinositol-3 kinase (PI3K), activation of the downstream kinase Akt and ultimately phosphorylation of IRF-3 [46] (Figure 1). The residue(s) phosphorylated by this Akt pathway is not Ser396 and remains to be identified. However, the phosphorylation has a key role in promoting the transactivation of IRF3 by facilitating its association with the co-activator CBP. In light of the study by Mori et al. [45], it would be interesting to determine if Ser386 is the residue phosphorylated by Akt.

Because many viruses produce dsRNA as part of their life cycle, the engagement of TLR3 by dsRNA and subsequent activation of IRF3 and induction of type IFNs is an important antiviral response in the host. However, other virally sensing systems exist that are independent of TLR3, yet activate IRF3 and induce IFNs. One such system is the cytoplasmic RNA helicase retinoic acid inducible gene I (RIG-I). It recognizes dsRNA and activates IRF3 by a mechanism independent of TRIF but probably dependent on TBK1 or IKK-i [47]. This is reviewed in more detail by Schröder and Bowie, also in this issue [31].

\section{Activation of IRF7 by TLR7 and TLR9}

Plasmacytoid DCs are specialized at producing extremely high levels of type I IFN in response to viral challenge (reviewed in Ref. [48]). They express TLR7 and TLR9 strongly and produce large amounts of IFN- $\alpha$ in response to the TLR7 ligand, single stranded viral RNA and TLR9 activators, such as DNA viruses and CpG oligonucleotides [49-52]. However, TLR7 and TLR9 are entirely dependent on Myd88 for signalling and do not use any of the other TIR domain-containing adaptors. Because the induction of type I IFNs is crucially dependent on the activation of IRFs, this raises the intriguing question of how these TLRs can activate IRFs without the help of TRIF. Plasmacytoid DCs show high levels of constitutive IRF7 expression, relative to monocyte-derived DCs [53], and two concomitant reports showed that Myd88 forms a complex with IRF7 to trigger its activation and induce IFN- $\alpha[54,55]$. TLR7 and TLR9 are located primarily in the intracellular endosomal compartment $[56,57]$ and the Myd88-IRF7 interaction takes place in the endosomal vesicles of the plasmacytoid DCs [58] (Figure 2). The complex must remain in the endosome for a prolonged time period and resist transfer to lysosomal vesicles to trigger activation of IRF7 [58]. This complex also contains IRAK4 and TRAF6 and the ubiquitin ligase activity of the latter is required for IRF activation [54,55]. Furthermore, TLR7 and TLR9 ligands activate IRF7 in a Myd88- and IRAK-4-dependent manner. A recent study showed that IRAK-1 interacts directly with, and phosphorylates, IRF7 [59] and TLR7 and TLR9 ligands are severely impaired in their ability to activate IRF7 and induce IFN- $\alpha$ in IRAK-1-deficient plasmacytoid DCs. Intriguingly, these cells show relatively normal responsiveness to TLR7 and TLR9 ligands, with respect to NF- $\mathrm{KB}$ activation and proinflammatory cytokine induction. Thus, the certainty of a role for IRAK-1 in all Myd88-dependent $\mathrm{NF}-\kappa \mathrm{B}$ activation has been shattered and instead a new role for IRAK-1 in mediating TLR7 or TLR9 activation of IRF7 is being championed.

It is unclear whether or not IRAK-1 is the direct kinase for IRF7. It was not possible to show endogenous phosphorylation of IRF7 by IRAK-1 [59]. It is probable that IRF7 activation by IRAK-1 is independent of TBK1 and IKK-I because plasmacytoid DCs derived from mice 


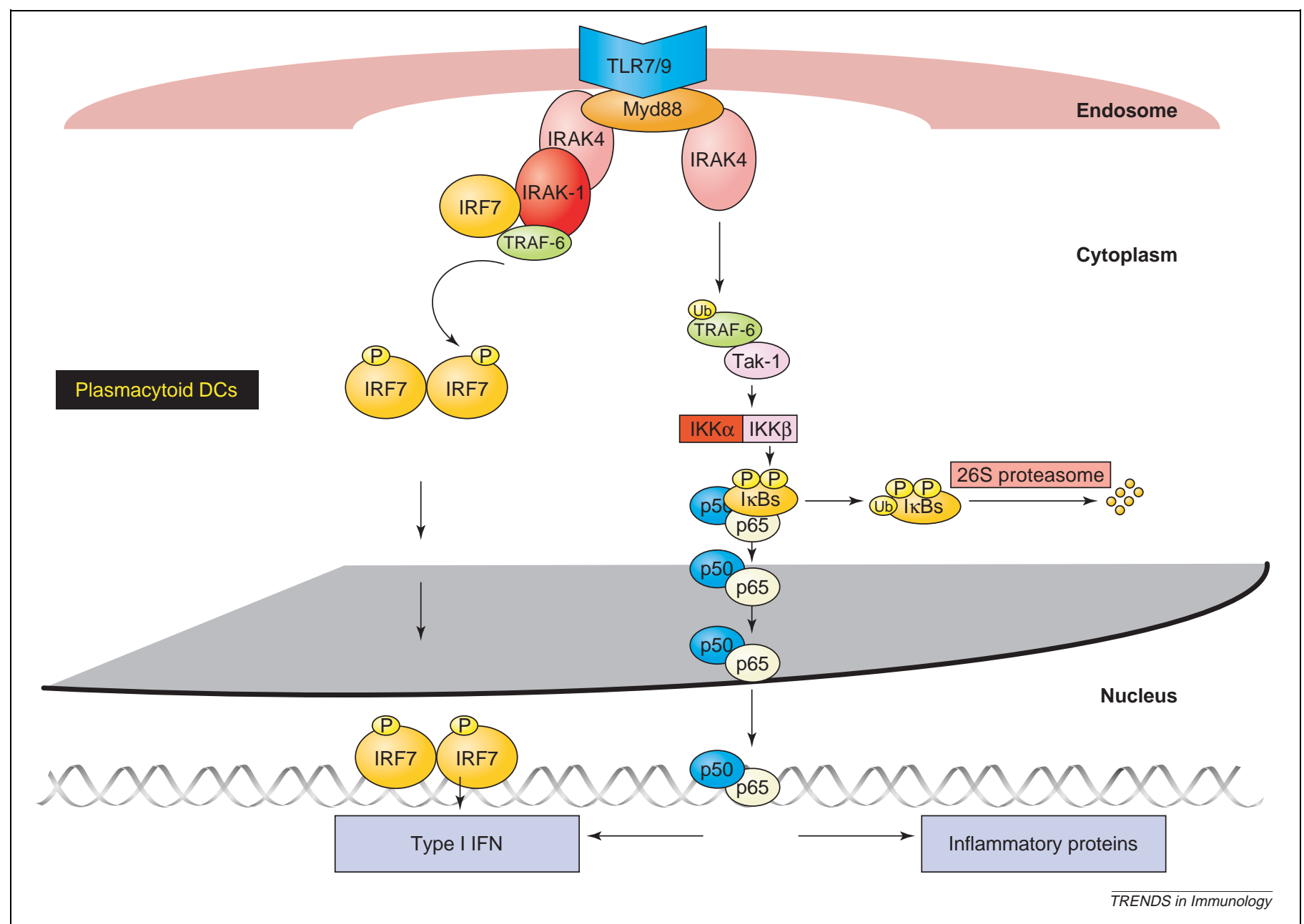

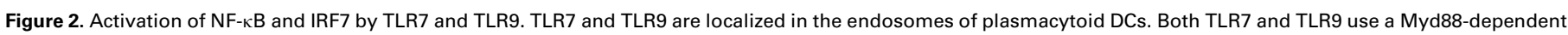

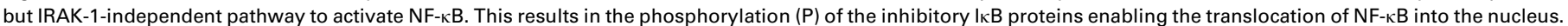

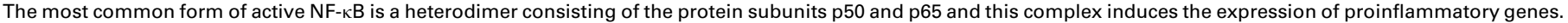
TLR7 and TLR9 use a Myd88- and IRAK-1-dependent pathway to phosphorylate IRF7, leading to its nuclear translocation and the induction of type I IFN genes.

deficient in TBK1 or IKK-i show normal responsiveness to TLR9 ligands with respect to induction of IFN- $\alpha$ [54]. However, this study does not exclude the possibility of functional redundancy between TBK1 and IKK-i. This can be addressed by assessing TLR9 induction of IFN- $\alpha$ in $I K K i^{-1-} T B K 1^{-1-}$ mice. Notably, the inability of Myd88 to activate TBK1 or IKK-i favours TBK1- or IKK-i-independent activation of IRF7 by IRAK-1.

Interestingly, although Myd88 interacts with, and activates, IRF7, it fails to interact with, or activate, IRF3. Thus, in plasmacytoid DCs, IRF7 is the key regulator of type I IFN production. This has been confirmed recently with the generation of mice deficient in the Irf7 gene [60]. Plasmacytoid DCs derived from these mice are completely non-responsive to TLR7 and TLR9 ligands with respect to induction of IFN- $\alpha$, whereas induction is normal in plasmacytoid DCs from IRF3-deficient mice. These findings indicate strongly that IRF7 is essential whereas IRF3 is dispensable for the induction of type I IFNs by TLR7 and TLR9 in plasmacytoid DCs.

\section{TLR signalling and IRF5}

A recent report has emphasized an important role for another member of the IRF family, IRF5, in TLR signalling [61]. The induction of proinflammatory cytokines, such as TNF, IL-6 and IL-12, by TLR3, TLR4, TLR5, TLR7 and TLR9 ligands is impaired severely in various cells from IRF5-deficient mice. By contrast, the induction of IFN- $\alpha$ by TLR9 in plasmacytoid DCs from these mice is unaffected. Takaoka et al. [61] propose that IRF5 is involved selectively in the induction of proinflammatory cytokines by virtually all TLRs and this is consistent with the increased resistance of $\operatorname{Irf5} 5^{-1-}$ mice to lethal shock induced by unmethylated DNA and LPS. Furthermore, putative IRF5-binding sites are identifiable in the promoters of several genes encoding proinflammatory cytokines. Because NF- $\kappa$ B sites are also present in these promoters and NF- $\kappa \mathrm{B}$ is universally activated by all TLRs, IRF5 and NF- $\kappa$ B might enjoy a close relationship in terms of conjoint activation and functional co-operativity at their target promoters.

IRF5 associates with both Myd88 and TRAF-6 and ligands for TLR4 and TLR9 promote nuclear translocation of IRF5 in a Myd88-dependent manner [61] (Figure 3). However, many of the downstream mediators of the IRF5 pathway await identification. Although IRF-5 is phosphorylated by TBK1 and IKK-i, this phosphorylation does not lead to IRF-5 nuclear translocation or activation [62]. 


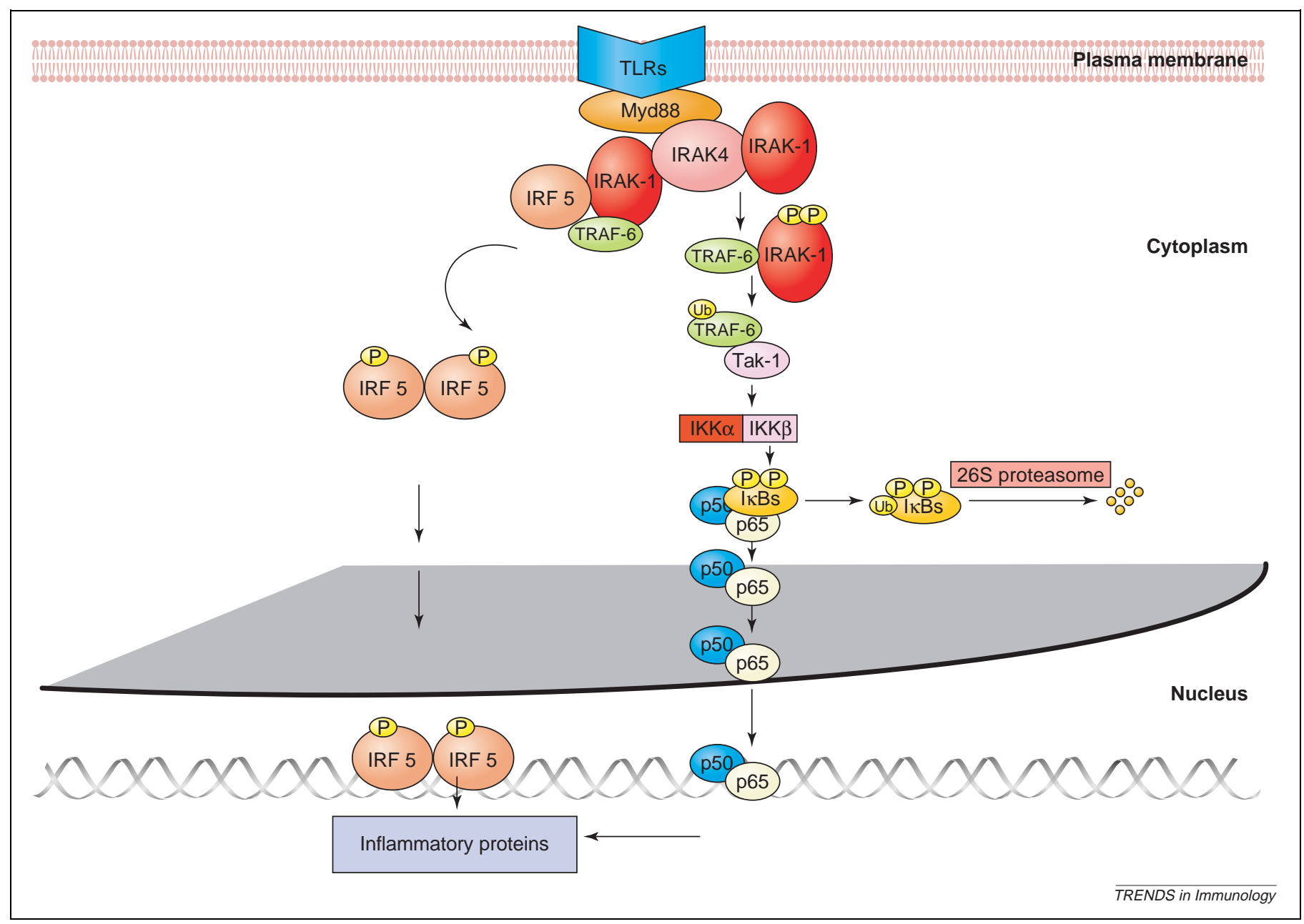

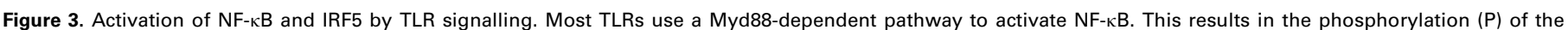

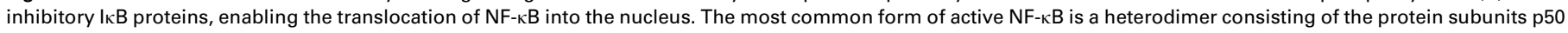

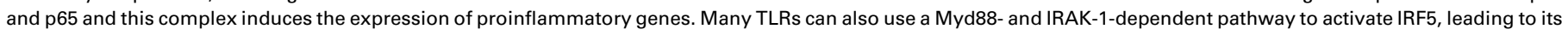
nuclear translocation and co-operation with NF- $\mathrm{KB}$ in the induction of proinflammatory genes.

The Myd88 activation of IRF-5 more closely resembles the pathway used by TLR7 and TLR9 in activating IRF7. Indeed, a recent report has shown an important role for IRAK-1 and TRAF-6 in TLR7 activation of IRF5 [63]. However, there are obvious differences between the Myd88 activation of IRF5 and IRF7. The former is promoted by most, if not all, TLRs, whereas the latter is more restricted to TLR7 and TLR9. Furthermore, the mechanism underlying TLR3 activation of IRF5 awaits characterisation. TLR3 does not use Myd88 and thus IRF5 might also be subject to regulation in a Myd88-independent manner. However, Schoenemeyer et al. [63] contends that TLR3 does not activate IRF5 and this controversy awaits further clarification.

\section{Concluding remarks}

The last two years have witnessed an enormous jump in our understanding of the role of IRFs in TLR biology and in the delineation of the TLR signal transduction pathways that regulate IRF activation. Many of the signalling molecules are shared with the NF- $\kappa$ B pathway and this is hardly surprising because the co-ordinated activation of IRF3, IRF7 and NF- $\mathrm{B}$ provides for maximal induction of type I IFNs, whereas conjoint activation of IRF5 and
NF- $\kappa$ B ensures functional co-operativity at the promoters of proinflammatory genes. Furthermore, it is becoming increasingly evident that different members of the IRF family are involved in controlling the expression of different profiles of genes by TLRs, and this might be crucial in ensuring the expression of appropriate gene profiles in response to specific pathogens. However, the molecular basis to this specificity is far from understood and many questions require addressing. Myd88 promotes activation of IRF7 and induction of type I IFN in response to TLR7 and TLR9; however, it is currently unclear why other TLRs, such as TLR2, which also use Myd88, fail to induce IFN.

Also, why have several signalling pathways evolved to activate the same IRF transcription factor? TLR3 and TLR4 use the TRIF-TBK1 (IKKi) axis, whereas TLR7 and TLR9 use the Myd88-IRAK4-IRAK-1-TRAF-6 cascade to activate IRF7. One plausible proposal is that the host uses two different sensing systems to detect different virus types but both culminate in a similar antiviral response. Regardless of the answers to these and other questions, it is clear that TLR regulation of IRFs is crucially important in regulating the expression of genes that mediate innate and adaptive immunity. Furthermore, this area offers 
much potential in terms of therapeutic exploitation. A recent report has indicated that TLR9-induced type I IFN is protective from colitis [64]. It is clear that the further clarification of the molecular basis of the activation of IRF members by TLRs is a worthwhile goal.

\section{Acknowledgements}

P.N.M. is funded by Health Research Board of Ireland, Science Foundation Ireland and Higher Education Authority of Ireland.

\section{References}

1 Martin, M.U. and Wesche, H. (2002) Summary and comparison of the signaling mechanisms of the Toll/interleukin-1 receptor family. Biochim. Biophys. Acta 1592, 265-280

2 Medzhitov, R. et al. (1998) MyD88 is an adaptor protein in the hToll/ IL-1 receptor family signaling pathways. Mol. Cell 2, 253-258

3 Burns, K. et al. (2000) Tollip, a new component of the IL-1RI pathway, links IRAK to the IL-1 receptor. Nat. Cell Biol. 2, 346-351

$4 \mathrm{Li}$, S. et al. (2002) IRAK-4: a novel member of the IRAK family with the properties of an IRAK-kinase. Proc. Natl. Acad. Sci. U. S. A. 99, $5567-5572$

5 Cao, Z. et al. (1996) IRAK: a kinase associated with the interleukin-1 receptor. Science $271,1128-1131$

6 Deng, L. et al. (2000) Activation of the IкB kinase complex by TRAF6 requires a dimeric ubiquitin-conjugating enzyme complex and a unique polyubiquitin chain. Cell 103, 351-361

7 Wang, C. et al. (2001) TAK1 is a ubiquitin-dependent kinase of MKK and IKK. Nature 412, 346-351

8 Ninomiya-Tsuji, J. et al. (1999) The kinase TAK1 can activate the NIK-I $\kappa \mathrm{B}$ as well as the MAP kinase cascade in the IL-1 signalling pathway. Nature 398, 252-256

$9 \mathrm{Yi}$, A.K. and Krieg, A.M. (1998) CpG DNA rescue from anti-IgMinduced WEHI-231 B lymphoma apoptosis via modulation of $\mathrm{I} \kappa \mathrm{B} \alpha$ and

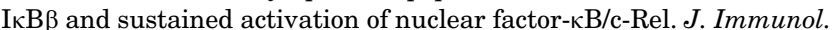
$160,1240-1245$

10 Medzhitov, R. et al. (1997) A human homologue of the Drosophila Toll protein signals activation of adaptive immunity. Nature 388, 394-397

11 O'Neill, L.A. (2002) Signal transduction pathways activated by the IL-1 receptor/Toll-like receptor superfamily. Curr. Top. Microbiol. Immunol. 270, 47-61

12 Kaisho, T. and Akira, S. (2001) Dendritic-cell function in Toll-like receptor- and MyD88-knockout mice. Trends Immunol. 22, 78-83

13 Kawai, T. et al. (1999) Unresponsiveness of MyD88-deficient mice to endotoxin. Immunity 11, 115-122

14 Kawai, T. et al. (2001) Lipopolysaccharide stimulates the MyD88independent pathway and results in activation of IFN-regulatory factor 3 and the expression of a subset of lipopolysaccharide-inducible genes. J. Immunol. 167, 5887-5894

15 Kaisho, T. et al. (2001) Endotoxin-induced maturation of MyD88deficient dendritic cells. J. Immunol. 166, 5688-5694

16 Fitzgerald, K.A. et al. (2001) Mal (MyD88-adapter-like) is required for Toll-like receptor-4 signal transduction. Nature 413, 78-83

17 Horng, T. et al. (2001) TIRAP: an adapter molecule in the Toll signaling pathway. Nat. Immunol. 2, 835-841

18 Horng, T. et al. (2002) The adaptor molecule TIRAP provides signalling specificity for Toll-like receptors. Nature 420, 329-333

19 Yamamoto, M. et al. (2002) Essential role for TIRAP in activation of the signalling cascade shared by TLR2 and TLR4. Nature 420 , 324-329

20 Yamamoto, M. et al. (2003) Role of adaptor TRIF in the MyD88independent Toll-like receptor signaling pathway. Science 301, 640-643

21 Hoebe, K. et al. (2003) Identification of Lps2 as a key transducer of MyD88-independent TIR signalling. Nature 424, 743-748

22 Oshiumi, H. et al. (2003) TICAM-1, an adaptor molecule that participates in Toll-like receptor 3-mediated interferon- $\beta$ induction. Nat. Immunol. 4, 161-167

23 Oshiumi, H. et al. (2003) TIR-containing adapter molecule (TICAM)-2, a bridging adapter recruiting to Toll-like receptor 4 TICAM-1 that induces interferon- $\beta$. J. Biol. Chem. 278, 49751-49762

24 Jiang, Z. et al. (2004) Toll-like receptor 3-mediated activation of NF-кB and IRF3 diverges at Toll-IL-1 receptor domain-containing adapter inducing IFN-B. Proc. Natl. Acad. Sci. U. S. A. 101, 3533-3538
25 Sato, S. et al. (2003) Toll/IL-1 receptor domain-containing adaptor inducing IFN- $\beta$ (TRIF) associates with TNF receptor-associated factor 6 and TANK-binding kinase 1 , and activates two distinct transcription factors, NF- $\mathrm{BB}$ and IFN-regulatory factor-3, in the Toll-like receptor signaling. J. Immunol. 171, 4304-4310

26 Gohda, J. et al. (2004) Cutting edge: TNFR-associated factor (TRAF) 6 is essential for MyD88-dependent pathway but not toll/IL-1 receptor domain-containing adaptor-inducing IFN- $\beta$ (TRIF)-dependent pathway in TLR signaling. J. Immunol. 173, 2913-2917

27 Meylan, E. et al. (2004) RIP1 is an essential mediator of Toll-like receptor 3-induced NF-kB activation. Nat. Immunol. 5, 503-507

28 Alexopoulou, L. et al. (2001) Recognition of double-stranded RNA and activation of NF- $\mathrm{BB}$ by Toll-like receptor 3. Nature 413, 732-738

29 Hoebe, K. and Beutler, B. (2004) LPS, dsRNA and the interferon bridge to adaptive immune responses: Trif, Tram, and other TIR adaptor proteins. J. Endotoxin Res. 10, 130-136

30 Taniguchi, T. et al. (2001) IRF family of transcription factors as regulators of host defense. Annu. Rev. Immunol. 19, 623-655

31 Schröder, M. and Bowie, A.G. TLR3 in antiviral immunity: key player or bystander? Trends Immunol. (in press)

32 Doyle, S. et al. (2002) IRF3 mediates a TLR3/TLR4-specific antiviral gene program. Immunity $17,251-263$

33 Servant, M.J. et al. (2003) Identification of the minimal phosphoacceptor site required for in vivo activation of interferon regulatory factor 3 in response to virus and double-stranded RNA. J. Biol. Chem. 278, 9441-9447

34 Fitzgerald, K.A. et al. (2003) IKK $\varepsilon$ and TBK1 are essential components of the IRF3 signaling pathway. Nat. Immunol. 4, 491-496

35 Sharma, S. et al. (2003) Triggering the interferon antiviral response through an IKK-related pathway. Science 300, 1148-1151

36 Tojima, Y. et al. (2000) NAK is an IкB kinase-activating kinase. Nature 404, 778-782

37 Bonnard, M. et al. (2000) Deficiency of T2K leads to apoptotic liver degeneration and impaired $\mathrm{NF}-\mathrm{\kappa B}$-dependent gene transcription. EMBO J. 19, 4976-4985

38 Sasai, M. et al. (2005) Cutting Edge: NF-кB-activating kinaseassociated protein 1 participates in TLR3/Toll-IL-1 homology domain-containing adapter molecule-1-mediated IFN regulatory factor 3 activation. J. Immunol. 174, 27-30

39 McWhirter, S.M. et al. (2004) IFN-regulatory factor 3-dependent gene expression is defective in Tbk1-deficient mouse embryonic fibroblasts. Proc. Natl. Acad. Sci. U. S. A. 101, 233-238

$40 \mathrm{Hemmi}$, H. et al. (2004) The roles of two I $\kappa$ B kinase-related kinases in lipopolysaccharide and double stranded RNA signaling and viral infection. J. Exp. Med. 199, 1641-1650

41 Perry, A.K. et al. (2004) Differential requirement for TANK-binding kinase-1 in type I interferon responses to toll-like receptor activation and viral infection. J. Exp. Med. 199, 1651-1658

42 Fitzgerald, K.A. et al. (2003) LPS-TLR4 signaling to IRF-3/7 and NF-кB involves the Toll adapters TRAM and TRIF. J. Exp. Med. 198, 1043-1055

43 Yamamoto, M. et al. (2003) TRAM is specifically involved in the Tolllike receptor 4-mediated MyD88-independent signaling pathway. Nat. Immunol. 4, 1144-1150

$44 \mathrm{Kim}$, T. et al. (2000) Signaling pathways to the assembly of an interferon- $\beta$ enhanceosome. Chemical genetic studies with a small molecule. J. Biol. Chem. 275, 16910-16917

45 Mori, M. et al. (2004) Identification of Ser-386 of interferon regulatory factor 3 as critical target for inducible phosphorylation that determines activation. J. Biol. Chem. 279, 9698-9702

46 Sarkar, S.N. et al. (2004) Novel roles of TLR3 tyrosine phosphorylation and PI3 kinase in double-stranded RNA signaling. Nat. Struct. Mol. Biol. 11, 1060-1067

47 Yoneyama, M. et al. (2004) The RNA helicase RIG-I has an essential function in double-stranded RNA-induced innate antiviral responses. Nat. Immunol. 5, 730-737

48 Liu, Y.J. (2005) IPC: Professional type 1 interferon-producing cells and plasmacytoid dendritic cell precursors. Annu. Rev. Immunol. 23, 275-306

49 Heil, F. et al. (2004) Species-specific recognition of single-stranded RNA via Toll-like receptor 7 and 8 . Science $303,1526-1529$ 
50 Diebold, S.S. et al. (2004) Innate antiviral responses by means of TLR7-mediated recognition of single-stranded RNA. Science 303, 1529-1531

51 Bauer, S. et al. (2001) Human TLR9 confers responsiveness to bacterial DNA via species-specific CpG motif recognition. Proc. Natl. Acad. Sci. U. S. A. 98, 9237-9242

52 Lund, J. et al. (2003) Toll-like receptor 9-mediated recognition of Herpes simplex virus-2 by plasmacytoid dendritic cells. J. Exp. Med. 198, 513-520

53 Izaguirre, A. et al. (2003) Comparative analysis of IRF and IFN- $\alpha$ expression in human plasmacytoid and monocyte-derived dendritic cells. J. Leukoc. Biol. 74, 1125-1138

54 Kawai, T. et al. (2004) Interferon- $\alpha$ induction through Toll-like receptors involves a direct interaction of IRF7 with MyD88 and TRAF6. Nat. Immunol. 5, 1061-1068

55 Honda, K. et al. (2004) Role of a transductional-transcriptional processor complex involving MyD88 and IRF-7 in Toll-like receptor signaling. Proc. Natl. Acad. Sci. U. S. A. 101, 15416-15421

56 Latz, E. et al. (2004) TLR9 signals after translocating from the ER to CpG DNA in the lysosome. Nat. Immunol. 5, 190-198
57 Ahmad-Nejad, P. et al. (2002) Bacterial CpG-DNA and lipopolysaccharides activate Toll-like receptors at distinct cellular compartments. Eur. J. Immunol. 32, 1958-1968

58 Honda, K. et al. (2005) Spatiotemporal regulation of MyD88-IRF-7 signalling for robust type-I interferon induction. Nature 434, 1035-1040

59 Uematsu, S. et al. (2005) Interleukin-1 receptor-associated kinase-1 plays an essential role for Toll-like receptor (TLR)7- and TLR9mediated interferon- $\alpha$ induction. J. Exp. Med. 201, 915-923

60 Honda, K. et al. (2005) IRF-7 is the master regulator of type-I interferon-dependent immune responses. Nature 434, 772-777

61 Takaoka, A. et al. (2005) Integral role of IRF-5 in the gene induction programme activated by Toll-like receptors. Nature 434, 243-249

62 Lin, R. et al. (2005) A CRM1-dependent nuclear export pathway is involved in the regulation of IRF-5 subcellular localization. J. Biol. Chem. 280, 3088-3095

63 Schoenemeyer, A. et al. (2005) The interferon regulatory factor, IRF5, is a central mediator of toll-like receptor 7 signaling. J. Biol. Chem. 280, 17005-17012

64 Katakura, K. et al. (2005) Toll-like receptor 9-induced type I IFN protects mice from experimental colitis. J. Clin. Invest. 115, 695-702

\section{Elsevier celebrates two anniversaries with gift to university libraries in the developing world}

In 1580, the Elzevir family began their printing and bookselling business in the Netherlands, publishing works by scholars such as John Locke, Galileo Galilei and Hugo Grotius. On 4 March 1880, Jacobus George Robbers founded the modern Elsevier company intending, just like the original Elzevir family, to reproduce fine editions of literary classics for the edification of others who shared his passion, other 'Elzevirians'. Robbers co-opted the Elzevir family's old printer's mark, visually stamping the new Elsevier products with a classic old symbol of the symbiotic relationship between publisher and scholar. Elsevier has since become a leader in the dissemination of scientific, technical and medical (STM) information, building a reputation for excellence in publishing, new product innovation and commitment to its STM communities.

In celebration of the House of Elzevir's 425th anniversary and the 125th anniversary of the modern Elsevier company, Elsevier will donate books to 10 university libraries in the developing world. Entitled 'A Book in Your Name', each of the 6700 Elsevier employees worldwide has been invited to select one of the chosen libraries to receive a book donated by Elsevier. The core gift collection contains the company's most important and widely used STM publications including Gray's Anatomy, Dorland's IIlustrated Medical Dictionary, Essential Medical Physiology, Cecil Essentials of Medicine, Mosby's Medical, Nursing and Allied Health Dictionary, The Vaccine Book, Fundamentals of Neuroscience, and Myles Textbook for Midwives.

The 10 beneficiary libraries are located in Africa, South America and Asia. They include the Library of the Sciences of the University of Sierra Leone; the library of the Muhimbili University College of Health Sciences of the University of Dar es Salaam, Tanzania; the library of the College of Medicine of the University of Malawi; and the libraries of the University of Zambia, Universite du Mali, Universidade Eduardo Mondlane, Mozambique; Makerere University, Uganda; Universidad San Francisco de Quito, Ecuador; Universidad Francisco Marroquin, Guatemala; and the National Centre for Scientific and Technological Information (NACESTI), Vietnam.

Through 'A Book in Your Name', the 10 libraries will receive approximately 700 books at a retail value of approximately 1 million US dollars.

$$
\text { For more information, visit www.elsevier.com }
$$

